\title{
Typical Hus: Evidence of Acute Phase Complement Activation from a Daycare Outbreak
}

\author{
Tammy M Brady ${ }^{1 *}$, Cozumel Pruette ${ }^{1}$, Lauren F Loeffler ${ }^{1}$, Darcy Weidemann ${ }^{2}$, John J Strouse ${ }^{3,4}$, Eleni \\ Gavriilaki $^{4}$ and Robert A Brodsky ${ }^{4}$ \\ ${ }^{1}$ Division of Pediatric Nephrology, Johns Hopkins University School of Medicine, USA \\ 2 Division of Pediatric Nephrology, Children's Mercy Hospital, Kansas City, MO, USA \\ ${ }^{3}$ Division of Pediatric Hematology Johns Hopkins University School of Medicine, USA \\ ${ }^{4}$ Division of Hematology, Johns Hopkins University School of Medicine, USA
}

${ }^{*}$ Corresponding author: Tammy M Brady, Division of Pediatric Nephrology, Johns Hopkins University School of Medicine, The David M. Rubenstein Child Health Building, 200 North Wolfe Street, Room 3057, Baltimore, MD 21287, USA, Tel: 410-955-2467; E-mail: tbrady8@jhmi.edu

Received date: April 15, 2016; Accepted date: May 03, 2016; Published date: May 06, 2016

Citation: Brady TM, Pruette C, Loeffler LF, Weidemann D, Strouse JJ, et al. (2016) Typical Hus: Evidence of Acute Phase Complement Activation from a Daycare Outbreak. J Clin Exp Nephrol 1: 11. DOI: 10.21767/2472-5056.100011

Copyright: (C) 2016 Brady TM, et al. This is an open-access article distributed under the terms of the Creative Commons Attribution License, which permits unrestricted use, distribution, and reproduction in any medium, provided the original author and source are credited.

\section{Abstract}

The clinical manifestations of typical hemolytic uremic syndrome (HUS) encompass a wide spectrum. Despite the potentially severe sequelae from this syndrome, treatment approaches remain supportive. We present the clinical course of a child who contracted Shiga toxin-positive $E$. coli (STEC) from a daycare center during an outbreak. Utilizing the modified Ham test which is a rapid, serum-based functional assay used to detect activation of the alternative pathway of complement as observed in atypical HUS, patient sera revealed evidence of increased complement activation in the acute phase of the syndrome but not after resolution. Further, this complement activation was attenuated by eculizumab in vitro, an effect that was replicated in vitro utilizing Shiga toxin as a stimulus of complement activation in normal serum. Our report suggests that complement blockade may be effective in the treatment of STEC-HUS when initiated early in the disease. Given the epidemic nature of the disease that limits the feasibility of randomized clinical trials, further studies are needed to determine the value of early eculizumab treatment in STEC-HUS.

Keywords: Hemolytic uremic syndrome; Hemolytic anemia; White blood cell

\section{Abbreviations:}

HD (hospital day); HUS (hemolytic uremic syndrome); PD (peritoneal dialysis); PRBC (packed red blood cell); STEC (Shiga toxin-positive E. coli); STX2 (Shiga toxin type 2); WBC (white blood cell).

\section{Introduction}

The clinical manifestations of Shiga toxin-positive E. coli hemolytic uremic syndrome (STEC-HUS) span a wide spectrum, with some children mildly affected with minimal symptoms to other children having a severe clinical course resulting in renal failure, substantial neurological consequences, and even death. The care of children with STEC-HUS remains supportive, with no therapies available to directly treat the underlying pathophysiology. Evidence from human $[1,2]$ and animal $[3,4]$ models have suggested that complement activation may play a role in the course of STEC-HUS, although this has not been fully characterized. Eculizumab, a C5 monoclonal antibody indicated for the treatment of atypical HUS (microangiopathic hemolytic anemia due to complement dysregulation as a result of acquired or genetic disorders [5]), has been used in STEC-HUS with reports suggesting some benefit $[6,7]$ and others stating no benefit $[8,9]$. In this report, we present the case of a child who contracted STEC from a daycare center during an outbreak. Sera provided for testing revealed evidence of increased complement activation in the acute phase of the syndrome.

\section{Case Report}

The child is a 28 month old previously healthy white male who developed bloody diarrhea after three days of fever, crampy abdominal pain, emesis, and non-bloody diarrhea. Initial evaluation was notable for white blood cell count (WBC) 14.8 $\mathrm{k} / \mathrm{mm}^{3}$, hemoglobin $13 \mathrm{~g} / \mathrm{dL}$, platelets $279 \mathrm{k} / \mathrm{mm} 3$, creatinine $0.3 \mathrm{mg} / \mathrm{dL}$ and a physical exam concerning for intussusception. Abdominal ultrasound was notable for colitis. During hospital admission, he received intravenous fluid and morphine for pain control. He became oliguric, with acute renal failure, anemia and thrombocytopenia noted on hospital day (HD) 3: creatinine 3.1 $\mathrm{mg} / \mathrm{dL}$, platelets $9 \mathrm{k} / \mathrm{mm} 3$, hemoglobin $5.8 \mathrm{~g} / \mathrm{dL}$. A peripheral blood smear revealed schistocytes and severely decreased 
platelets, consistent with a microangiopathic hemolytic anemia. His renal failure progressed and peritoneal dialysis (PD) was initiated on HD 5 and continued for seven days. His peak creatinine was $5.7 \mathrm{mg} / \mathrm{dL}$, which decreased to $0.6 \mathrm{mg} / \mathrm{dL}$ by discharge (HD 15). He required six packed red blood cell (PRBC) transfusions to maintain hemoglobin $>6 \mathrm{~g} / \mathrm{dL}$ and received two platelet transfusions, each prior to a procedure. Three days after discharge his hemoglobin was $12 \mathrm{~g} / \mathrm{dL}$, platelets $350 \mathrm{k} / \mathrm{mm} 3$, creatinine $0.37 \mathrm{mg} / \mathrm{dL}$.

He had no neurological sequelae related to underlying HUS. He had significant anorexia/nausea throughout the hospitalization and received nasogastric tube feeds until discharge. Stool cultures at the state laboratory were positive for Shiga toxin type 2 (STX2) consistent with likely E. coli. An outbreak of infectious diarrhea and HUS was subsequently noted at the patient's daycare, and the local Health Department was closely involved.

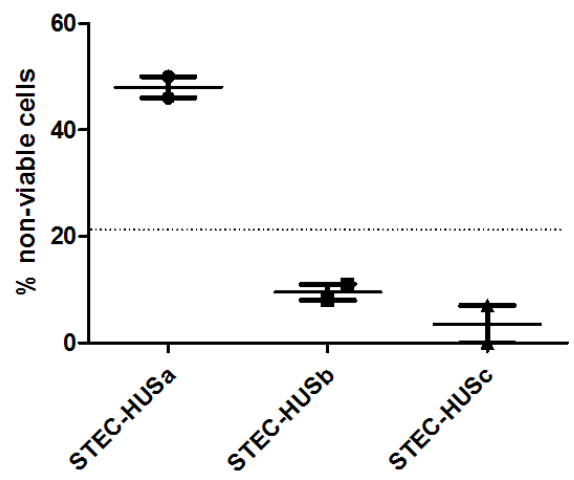

Figure 1: A): Complement activation in Shiga-toxin associated HUS- Increased percentage of non-viable cells in the modified Ham test was observed in the acute phase of Shiga-toxin associated HUS (STEC-HUSa) The same patient was tested twice after resolution of the syndrome ( 4 and 7 days after the first sample, symbolized as STEC-HUSb and c), showing normalization of cell killing. The dotted line symbolizes the cut-off value ( $21.5 \%$ non-viable cells) above which percentage of non-viable cells suggests increased complement activation observed in atypical HUS. Results from two independent experiments are shown.

Further laboratory testing was performed after written informed consent (see Supplementary Methods). Three samples was provided: one in the acute phase of the syndrome and two after the resolution of the syndrome. We utilized the modified Ham test that has been recently described as a functional assay able to detect activation of the alternative pathway of complement observed in complement-mediated hemolytic anemias $[10,11]$. The test was performed as previously described. Briefly, this rapid and simple cell proliferation assay detects complement-mediated cell killing in the patient serum. As shown in Figure $1 \mathrm{~A}$, the acute phase sample caused increased cell killing in the modified Ham test, while the two samples during remission showed normalization of cell killing. These findings suggest that increased complement activation is evident in the acute phase of the disease. To further investigate these findings, we first sought to determine whether complement inhibition with eculizumab nullifies the effects of acute STECHUS serum. Therefore, we incubated STEC-HUS serum with serum containing eculizumab at different ratios and tested its effect on the modified Ham test. Indeed, addition of eculizumab containing serum resulted in a normalization of the modified Ham test results in all ratios (Figure 1B).

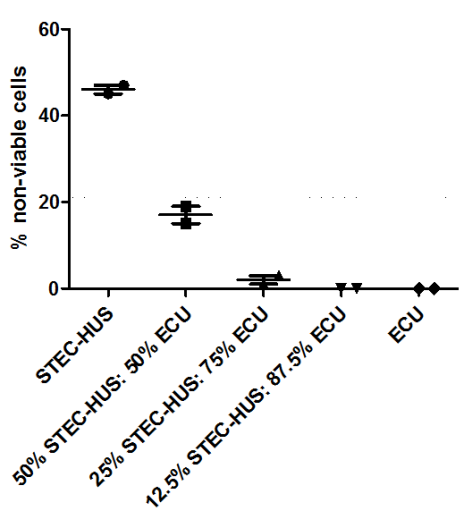

Figure 1: B): Complement activation in Shiga-toxin associated HUS- Eculizumab containing serum (ECU) was collected within 60 minutes of eculizumab infusion from a PNH patient. ECU was mixed with serum from the acute STEC-HUS in different percentages (50-50\%, $25-75 \%$ and $12.5-87.5 \%$ of STEC-HUS and ECU sera respectively). Total amount of serum in the assay remained unchanged (20\%). Eculizumab containing serum resulted in a normalization of the modified Ham test results in all ratios. Results from two independent experiments are shown.

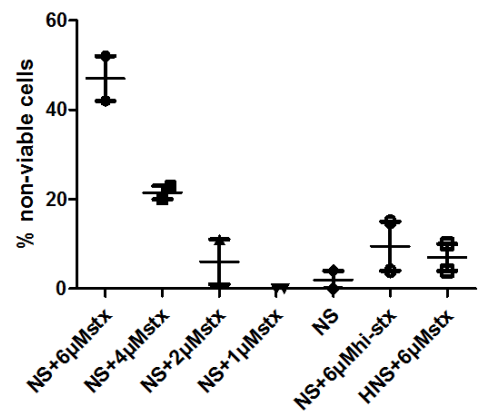

Figure 1: C): Complement activation in Shiga-toxin associated HUS- Recombinant shiga-toxin 2 from E.coli was added in normal serum (NS) to replicate the effects of STEC-HUS. Addition of shiga-toxin (stx) resulted in increased percentage of non-viable cells compared to normal serum alone and with heat-inactivated shiga-toxin (hi-stx), as well as heatinactivated normal serum (HNS) with maximum amount of shiga-toxin. Results from two independent experiments are shown.

Then, we tried to mimic the effect caused by STEC-HUS serum utilizing normal serum with Shiga toxin. Recombinant STX2 from 
E.coli was added in normal serum. Addition of Shiga toxin in normal serum resulted in an increase in cell killing (Figure 1C), similar to that caused by STEC-HUS serum. To exclude the possibility of direct Shiga toxin mediated cell lysis, we also tested the effect of Shiga toxin added in complement-inactivated normal serum, which caused no increased in cell killing.

\section{Discussion}

Our report presents the clinical course of a child with STECHUS who contracted STEC during a daycare outbreak. He had laboratory findings of increased complement activation in the acute phase of the disease but not after the resolution of the syndrome. In addition, we are able to show that addition of Shiga toxin in normal serum mimics the increased complement activation seen with STEC-HUS serum. More importantly, STECHUS serum is responsive to complement blockade by eculizumab in vitro.

Reports on eculizumab treatment in STEC-HUS have been conflicting. In line with our experimental data, initial and later case reports have shown efficacy of early treatment with eculizumab in severe cases of STEC-HUS [6,7]. However, analyses of the German outbreak and registry have not favored the use of eculizumab over other treatment options $[8,9]$. These analyses are limited by their retrospective nature and lack of randomization that resulted in a comparison between different treatment groups with different disease severities. Our in vitro data suggests that activation of the APC plays a role in the endorgan damage of STEC-HUS. It does not prove that eculizumab would alter the natural history of this disease but suggest that if eculizumab therapy is attempted in future clinical trials, it should be administered early in the disease process.

Another interesting observation of these studies was the benefits of antibiotic treatment that resulted in a shorter duration of E.coli excretion. Shiga toxin attacks cells expressing globotriosylceramide (Gb3) receptors, such as renal endothelial cells. It has multiple cellular effects that ultimately lead to cellular damage and apoptosis [12]. Several experimental studies have also linked Shiga toxin with complement activation in vitro. Indeed, shiga toxin inhibits major complement regulators, complement Factor $\mathrm{H}(\mathrm{CFH})$ and $\mathrm{CFH}$-related proteins, mimicking effects caused by the loss-of-function mutations observed in atypical HUS $[2,13,14]$. Shiga toxin also reduces the expression of other complement regulators, such CD59 and thrombomodulin $[15,16]$. In addition, shiga toxin seems to directly activate complement component C3 and subsequently, the alternative pathway of complement [17]. Complement activation by shiga toxin has been also suggested by several murine models $[3,18,19]$. Recently, shiga toxin has also been shown to promote podocyte injury via the alternative complement pathway in an experimental HUS model [20]. These findings correspond to our experimental data showing that the presence of Shiga toxin causes a dose-dependent activation of complement in vitro.

In summary, our report suggests that shiga toxin is directly responsible for activating the alternative pathway of complement. Given the epidemic nature of HUS that limits the feasibility of randomized trials, further clinical studies are needed to evaluate early eculizumab treatment in STEC-HUS.

\section{Conflicts of Interest}

Robert Brodsky serves on the Scientific Advisory Board for Alexion Pharmaceuticals and Apellis Pharmaceuticals. None of the other authors have any conflicts of interest to disclose.

\section{Sources of Support:}

TMB is funded by the NIH/NHLBI (1K23 HL119622-01).

\section{References:}

1. Thurman JM, Marians R, Emlen W (2009) Alternative pathway of complement in children with diarrhea-associated hemolytic uremic syndrome. Clinical journal of the American Society of Nephrology: CJASN 4: 1920-1924.

2. Orth $D$, Khan AB, Naim A (2009) Shiga toxin activates complement and binds factor $\mathrm{H}$ : evidence for an active role of complement in hemolytic uremic syndrome. J Immunol 182: 6394-6400.

3. Keepers TR, Psotka MA, Gross LK, Obrig TG (2006) A murine model of HUS: Shiga toxin with lipopolysaccharide mimics the renal damage and physiologic response of human disease. Journal of the American Society of Nephrology: JASN 17: 3404-3414.

4. Zoja C, Locatelli M, Pagani C (2012) Lack of the lectin-like domain of thrombomodulin worsens Shiga toxin-associated hemolytic uremic syndrome in mice. J Immunol 189: 3661-3668.

5. Brodsky RA (2015) Complement in hemolytic anemia. Blood 126: 2459-2465.

6. Dinh A (2015) Anathasayanan A and Rubin LM: Safe and effective use of eculizumab in the treatment of severe Shiga toxin Escherichia coli-associated hemolytic uremic syndrome. American journal of health-system pharmacy: AJHP: official journal of the American Society of Health-System Pharmacists 72: 117-120.

7. Lapeyraque AL, Malina M, Fremeaux-Bacchi V (2011) Eculizumab in severe Shiga-toxin-associated HUS. The New England journal of medicine 364: 2561-2563.

8. Menne J, Nitschke M, Stingele R (2012) Validation of treatment strategies for enterohaemorrhagic Escherichia coli O104:H4 induced haemolytic uraemic syndrome: case-control study. BMJ 345: e4565.

9. Kielstein JT, Beutel G, Fleig S (2012) Best supportive care and therapeutic plasma exchange with or without eculizumab in Shigatoxin-producing E. coli 0104:H4 induced haemolytic-uraemic syndrome: an analysis of the German STEC-HUS registry. Nephrology, dialysis, transplantation: official publication of the European Dialysis and Transplant Association - European Renal Association 27: 3807-3815

10. Gavriilaki E, Yuan X, Ye Z (2015) Modified Ham test for atypical hemolytic uremic syndrome. Blood 125: 3637-3646.

11. Vaught AJ, Gavriilaki E, Hueppchen N (2016) Direct evidence of complement activation in HELLP syndrome: A link to atypical hemolytic uremic syndrome. Exp Hematol 44: 390-398.

12. Bauwens A, Betz J, Meisen I, Kemper B, Karch H, et al. (2013) Facing glycosphingolipid-Shiga toxin interaction: dire straits for endothelial cells of the human vasculature. Cellular and molecular life sciences : CMLS 70: 425-457. 
13. Poolpol K, Orth-Holler D, Speth C (2014) Interaction of Shiga toxin 2 with complement regulators of the factor $\mathrm{H}$ protein family. Molecular immunology 58: 77-84.

14. Pickering MC, de Jorge EG, Martinez-Barricarte R (2007) Spontaneous hemolytic uremic syndrome triggered by complement factor $\mathrm{H}$ lacking surface recognition domains. The Journal of experimental medicine 204: 1249-1256.

15. Ehrlenbach S, Rosales A, Posch W (2013) Shiga toxin 2 reduces complement inhibitor CD59 expression on human renal tubular epithelial and glomerular endothelial cells. Infection and immunity 81: 2678-2685.

16. Fernandez GC, Te Loo MW, van der Velden TJ, van der Heuvel LP, Palermo MS, et al. (2003) Decrease of thrombomodulin contributes to the procoagulant state of endothelium in hemolytic uremic syndrome. Pediatr Nephrol 18: 1066-1068.
17. Morigi M, Galbusera M, Gastoldi S (2011) Alternative pathway activation of complement by Shiga toxin promotes exuberant C3a formation that triggers microvascular thrombosis. J Immunol 187: 172-180.

18. Paixao-Cavalcante D, Botto M, Cook HT, Pickering MC (2009) Shiga toxin-2 results in renal tubular injury but not thrombotic microangiopathy in heterozygous factor $\mathrm{H}$-deficient mice. Clinical and experimental immunology 155: 339-347.

19. Barrett TJ, Potter ME, Strockbine NA (1990) Evidence for participation of the macrophage in Shiga-like toxin II-induced lethality in mice. Microbial pathogenesis 9: 95-103.

20. Locatelli M, Buelli S, Pezzotta A (2014) Shiga toxin promotes podocyte injury in experimental hemolytic uremic syndrome via activation of the alternative pathway of complement. Journal of the American Society of Nephrology : JASN 25: 1786-1798. 